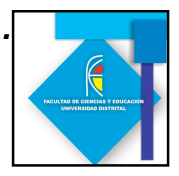

\title{
RECONTEXTUALIZACIÓN DEL PRINCIPIO DE CONSERVACIÓN DE LA ENERGÍA A TRAVÉS DE LA TEORÍA DE SISTEMAS.
}

\section{RECONTEXTUALIZATION OFTHE ENERGY CONSERVATION THR OUGH THE THEORY OF SYSTEMS.}

\author{
Dany Esteban Gallego Quiceno ${ }^{1}$
}

\section{Resumen}

En el contexto de la enseñanza de la física se abordan diferentes temáticas afines al principio de conservación de la energía que muestran dificultades conceptuales y procedimentales en donde se habla indistintamente de trabajo, calor y temperatura. Este hecho además de propiciar la confusión de los conceptos, refleja una particular manera de asumir los procesos de formalización en su relación con la organización de los fenómenos físicos: en tales procesos formalizar se reduce al uso de algoritmos y simbolismos matemáticos.

Buscando rutas alternativas para la enseñanza de los fenómenos termodinámicos, la presente investigación se desarrolla a partir de una reflexión histórico-epistemológica del PCE basada en la teoría de sistemas; se realiza un análisis histórico epistemológico de la manera como Newton, Mayer, Helmholtz, Robert Boyle, Joseph Black, formalizan estos conceptos, de tal manera que se recontextualizan y se formalizan en el contexto de la enseñanza. Se muestra con éste análisis como la resignificación del principio posibilita una mejor comprensión de los fenómenos termodinámicos en estudiantes de nivel universitario y de secundaria.

Palabras clave: Formalizar, Calor, Temperatura, Trabajo, Principio de Conservación de la Energía.

\begin{abstract}
In the context of physics education covers various topics related to the principle of conservation of energy that conceptual and procedural show where people referring to work, heat and temperature. This will also encourage the confusion of concepts, reflects a particular way of taking the process of formalizing their relationship with the organization of physical phenomena in such processes is reduced to formalize the use of algorithms and mathematical symbols.
\end{abstract}

\footnotetext{
${ }^{1}$ Licenciado en Matemáticas y Física, Docente Facultad de Educación, Universidad de Antioquia, Docente Colegio San José de las Vegas, Colombia. danygallego@yahoo.com
} 


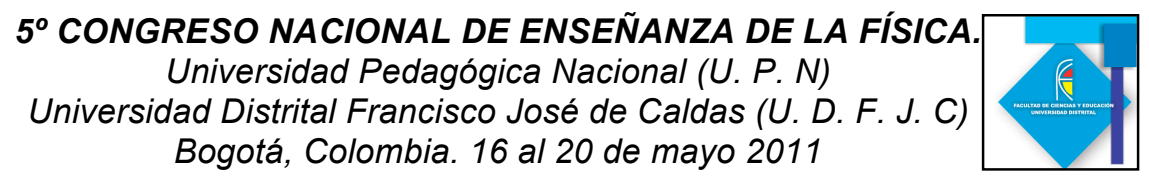

Looking for alternative routes to teaching thermodynamic phenomena, this research is developed from a historical-epistemological reflection of PCE based on systems theory, is a historical analysis of how epistemological as Newton, Mayer, Helmholtz, Robert Boyle, Joseph Black, formalize these concepts, so are recontextualized and formalized in the context of education. Shown with this analysis as the redefinition of the principle allows for a better understanding of the thermodynamic phenomena in students of college and high school level.

Keywords: Formalize, Heat, Temperature, Work, Law of Conservation of Energy.

\section{Introducción}

El siguiente artículo muestra los resultados de una investigación cualitativa por medio de un estudio intrínseco de casos realizado a estudiantes de secundaria (4 casos) y de nivel universitario(4 casos) por medio de los cuales se encontró que en el contexto de la enseñanza se evidencian diferentes dificultades conceptuales referidas a la manera en que se asume el principio de conservación de la energía; en ocasiones, se atribuye la dificultad del aprendizaje del PCE a las ideas previas de los estudiantes, debido a que éstos dotan a la energía de aspectos materiales al considerarla como una sustancia (Duit, 1987; Solomon, 1985) originando poca diferenciación entre el conocimiento científico y el conocimiento cotidiano (Duit, 1981; Solomon, 1983) en los que el significado de la energía es diferente.

Sin embargo, no todas las dificultades conceptuales referidas a los procesos de enseñanza-aprendizaje de la energía, se originan en las concepciones de los estudiantes, ya que en ocasiones no se consideran los sistemas energéticos sino que por el contrario se da un tratamiento netamente algorítmico o procedimental (Driver, 1985; López Gay, 1987) y por tanto no se realiza un adecuado tratamiento a las 4 características fundamentales de la energía (transformación, conservación, transferencia y degradación) (Duit, 1981, 1984).

Debido a las dificultades mostradas anteriormente y con la intención de plantear alternativas de enseñanza se aborda la perspectiva de sistemas y se hace un análisis histórico epistemológico de la manera como se formaliza el PCE.

\section{La teoría de Sistemas}

Desde la perspectiva de Guidoni, se considera que ver por sistemas es discretizar el espacio según criterios de estabilidad y coherencia, donde se rompe con la continuidad del universo y se consideran elementos, relaciones, estructuras de relaciones e interacciones. (Guidoni y Arca, 1990, p.5); de este modo, cuando se busca construir relaciones entre sistemas o entre partes de un sistema, es necesario determinar las variables que describen y representan explícitamente la interacción en forma definida.

Desde esta perspectiva, la energía se considera entonces una variable de proceso que da cuenta de una interacción entre sistemas, que a su vez permite asociarlo con las interacciones que producen, en los sistemas, las transformaciones. 


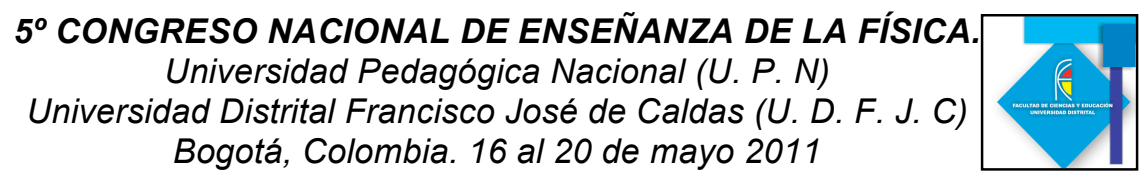

En este sentido se puede decir que las transformaciones o cambios de estado en un sistema son la evidencia de las interacciones entre sistemas; en el caso de los planteamientos de Mayer las interacciones son de orden mecánico o de orden térmico, desde aquí se posibilita analizar sistemas en los que se producen equilibraciones tal es el caso donde el principio de la conservación de la energía es visto como una variable de estado, que permite dar cuenta de la condición en la que se encuentra un sistema, o en su defecto el restablecimiento del equilibrio energético.

\section{Propuesta para enseñar el principio de conservación de energía.}

Entendiendo que la historia y la epistemología de la física es una tentativa para reorganizar y recontextualizar fenómenos físicos, se propone dentro de esta investigación un análisis de la manera como Newton, Mayer, Helmholtz, Robert Boyle, Joseph Black, formalizan estos conceptos, de tal manera que se recontextualizan y se formalizan en el contexto de la enseñanza.

De este modo y a raíz de las necesidades inherentes en el contexto de la enseñanza, para construir rutas conceptuales alternativas de los diferentes fenómenos físicos, se plantea una propuesta diferente para organizar y recontextualizar los diferentes fenómenos físicos, se proponen reflexiones histórico-epistemológicas, que posibilitan no sólo un análisis conceptual desde los problemas originales del conocimiento científico, sino que además posibilita significar a la Física como una disciplina históricamente construida e ideada por hombres.

Estos planteamientos se justifican porque se considera que la historia y la epistemología en la enseñanza: Motiva y despierta el interés por la ciencia; proporciona una mejor comprensión de los conceptos científicos mostrando su desarrollo y dinámica de construcción; propicia la comprensión de cómo se generan y validan los diferentes productos de la actividad científica; permite establecer relaciones entre los contenidos científicos y los intereses éticos, culturales y políticos de los contextos donde se produjeron (Matthews, 1994).

En esta perspectiva el hombre busca establecer relaciones con su entorno, entendiendo que desde este punto de vista, el conocimiento científico consiste en la búsqueda de significados de la realidad construida por el hombre, o como lo diría Elkana(1994), la ciencia está construida históricamente y está sometida a estándares de juicio históricamente definidos, teniendo en cuenta que el problema del conocimiento científico está centrado en la creación de condiciones para que sucedan los eventos, y se legitimen las postulaciones a través del consenso.

Como parte del análisis histórico-epistemológico que se propone se realiza la siguiente intervención: Se propone un acercamiento al principio a partir de la lectura de textos originales escritos por Newton, Mayer, Helmholtz, Robert Boyle, Joseph Black, en los dos últimos autores, se brinda un acercamiento desde la investigación realizada anteriormente sobre "los conceptos de calor y temperatura desde la perspectiva de sistemas" en donde se concluye que el calor es una variable de proceso que da cuenta de una interacción 


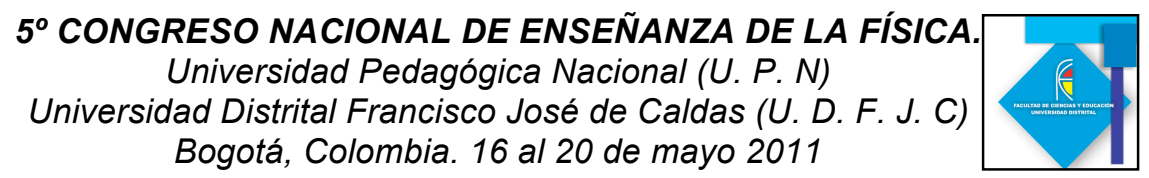

térmica y la temperatura es una variable de estado que da cuenta del estar o condición en el que se encuentra un sistema termodinámico; dando continuidad a la investigación se realiza un estudio complementario desde la perspectiva de Mayer donde se considera, a partir del análisis de textos originales, que:

En 1842, en su trabajo titulado Observaciones sobre las energías de la naturaleza inorgánica, Mayer no encuentra en términos cualitativos una equivalencia entre energía y movimiento, estando en contra pie a los planteamientos ya formulados por Rumford y Davy, quienes establecieron la equivalencia entre calor y movimiento; por su parte Mayer con su característica filosofía, establece que:

"En innumerables casos vemos que el movimiento cesa sin haber causado otro movimiento $o$ elevado un peso, pero la energía, una vez que existe, no puede ser aniquilada, solamente puede cambiar su forma; y entonces surge la pregunta: ¿qué otras formas es capaz de tomar la energía, aparte de las que ya conocemos?" " (Mayer, 1842).

Siguiendo con sus razonamientos cualitativos, se posibilita el establecimiento de la primera manifestación de la conservación y equivalencia de todas las energías, cuando Mayer afirma que

"Si las energías potencial y cinética son equivalentes al calor, éste también debe ser, de manera natural, equivalente a la energía cinética" " (Mayer, 1842).

De éste modo se propone entonces una deducción de la equivalencia entre calor, trabajo y las demás formas de energía diferente a la mostrada por Rumford, se toma distancia de la solución algorítmica de los problemas energéticos y se da una propuesta discursiva para la comprensión de dicho fenómeno físico.

De este modo es preciso afirmar entonces que el PCE visto desde la teoría de sistemas representa una variable de estado, que permite dar cuenta de la condición en la que se encuentra un sistema, o en su defecto el restablecimiento del equilibrio energético.

\section{Resultados obtenidos en la intervención.}

La propuesta alternativa de enseñanza-aprendizaje del principio de conservación de la energía aplicado a los estudiantes de secundaria (4 casos) y de nivel universitario (4 casos), evidenció como los estudios históricos epistemológicos enfocados a solucionar problemas en el contexto de la enseñanza, permiten despertar en los estudiantes un alto grado de interés por la construcción humana de la ciencia, de tal manera que esta tentativa proporciona una mejor comprensión de los conceptos científicos en el que se muestra su desarrollo y dinámica de construcción; adicional a esto, los estudios históricoepistemológicos propician la comprensión de cómo se generan y validan los diferentes productos de la actividad científica permitiendo establecer relaciones entre los contenidos científicos y los intereses éticos, culturales y políticos de los contextos donde se produjeron (Matthews, 1994), de este modo, se llevo a cabo un estudio de las bases 


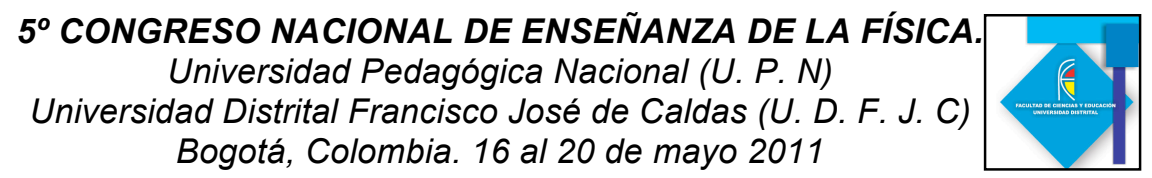

conceptuales y los principios inmersos en la ciencia; esta tentativa le brinda al maestro elementos válidos para la indagación sobre los procesos del conocimiento científico y a los estudiantes comprender el devenir histórico, socio cultural y el bien cultural de los planteamientos científicos.

\section{Referencias Bibliográficas.}

Arca, M; Guidoni, P.(1990) Enseñar Ciencia. Barcelona: Paidos Educador. p. 174.

Duit, R, (1981). Understanding Energy as a Conserved Quantity-Remarks, en R. U. Sexl. European Journal of Science Education, 3(3), pp. 291-301.

Duit, R. (1984). Learning the energy concept in school-empirical results from The Philippines and West Germany. Physics Education, 19, pp. 59-66

Duit, R. (1987). Should energy be illustrated as something quasi-material? European Journal of Science Education, 9(2), pp. 139-145.

Elkana, Y. (1983) "La ciencia como sistema cultural: Una aproximación Antropológica." Bogotá, Vol. 3, p. 65-80.

Gallego, D. Aguilar, Y. La formalización de los conceptos de calor y temperatura desde la perspectiva de sistemas, En: Colombia. 2009. Evento: X Conferencia interamericana de educación en física. Ponencia.

López, R. (1987). Las representaciones de los alumnos como punto de partida. El caso de la energía. Investigación en la Escuela, 3, pp. 47-54.

Solomon, J. (1985). Teaching the conservation of energy. Physics Education, 20, (pp. 165176). 